\title{
Hollow core dislocations in GaN
}

\section{Cherns}

\section{H.H. Wills Physics Laboratory, University of Bristol, Tyndall Avenue, Bristol BS8 1TL, UK}

Over the last 50 years, TEM studies of the core structure of dislocations have shown that dislocations often dissociate into partials, and that this dissociation can usually be explained by the associated reduction in the strain field energy. However, in recent years, it has become clear that dislocations in a number of materials, such as hexagonal GaN, can display hollow cores (Figure 1). In principle, this can also be explained by energy reduction, as proposed by Frank in 1952 [1], who argued that removal of the core material will release strain field energy at the expense of energy associated with the newly created surfaces. Frank showed that the equilibrium radius of the hollow core, $r=\mu b^{2} / 8 \pi^{2} \gamma$, where $\mu, b, \gamma$ are the shear modulus, Burgers vector and surface energy.

This paper examines the formation of hollow core dislocations in (0001)GaN films. These films normally contain 3 types of dislocation with Burgers vectors of $a$ (edge orientation, $b=0.32 \mathrm{~nm}$ ), $c$ (screw, $b=0.52 \mathrm{~nm})$ and $c+a($ mixed, $b=0.61 \mathrm{~nm})$, where $a, c$ are the unit cell parameters. In undoped GaN films grown by metal-organic chemical vapour deposition (MOCVD) or by hydride vapour phase epitaxy (HVPE), $c$-dislocations have been reported as hollow core type $(r=20-50 \mathrm{~nm})$, whereas $a$ and $c+a$ dislocations appear undissociated (e.g see [2]]. However, in MOCVD GaN pdoped with $\mathrm{Mg}, a$ and $c+a$ dislocations have been found to have hollow cores $(r=2-3 \mathrm{~nm})$ [3]. The situation is different again in GaN films grown by molecular beam epitaxy under Ga-rich conditions, where only $c+a$ dislocations have been reported as open core [4]. These conflicting results show little support for Frank's theory: there is no correlation between hollow core formation and the magnitude of $b$, and estimated values for $r$ are much too small (1nm or less [2]) to fit experimental results. Instead we believe that segregation to dislocations is an important factor. This has been confirmed by recent STEM studies of HVPE-grown GaN, which show evidence for substantial segregation of oxygen to dislocation cores [5]. This is illustrated in Figure 2, which compares background-stripped EELS line profiles for the $\mathrm{Ga}_{2,3}, \mathrm{~N}-\mathrm{K}$ and $\mathrm{O}-\mathrm{K}$ edge signals across a hollow core c-dislocation viewed end-on. The profiles have been scaled to show relative atomic ratios for $\mathrm{Ga}, \mathrm{N}$ and $\mathrm{O}$. In total it is estimated that up to two surface monolayers of nitrogen have been replaced by oxygen.

Results such as those in Figure 2 suggest that the formation of hollow core dislocations is intimately associated with impurity segregation. The growth method is clearly also important. In the paper, the various observations will be reviewed, and a number of competing mechanisms for hollow core formation will be presented. The significance of the results for understanding dislocations in other systems will be discussed.

References

[1] F.C. Frank, Acta Cryst. 4, 497 (1951)

[2] D. Cherns et al, J. Cryst. Growth 178, 201-206 (1997)

[3] D.Cherns et al, Appl. Phys. Lett 81, 4541-4543 (2002)

[4] M.Q. Baines et al, MRS Proc. Vol 743 Paper L2.5 (2002)

[5] M.E. Hawkridge and D. Cherns, Appl. Phys. Lett. 87, 221903 (2005) 

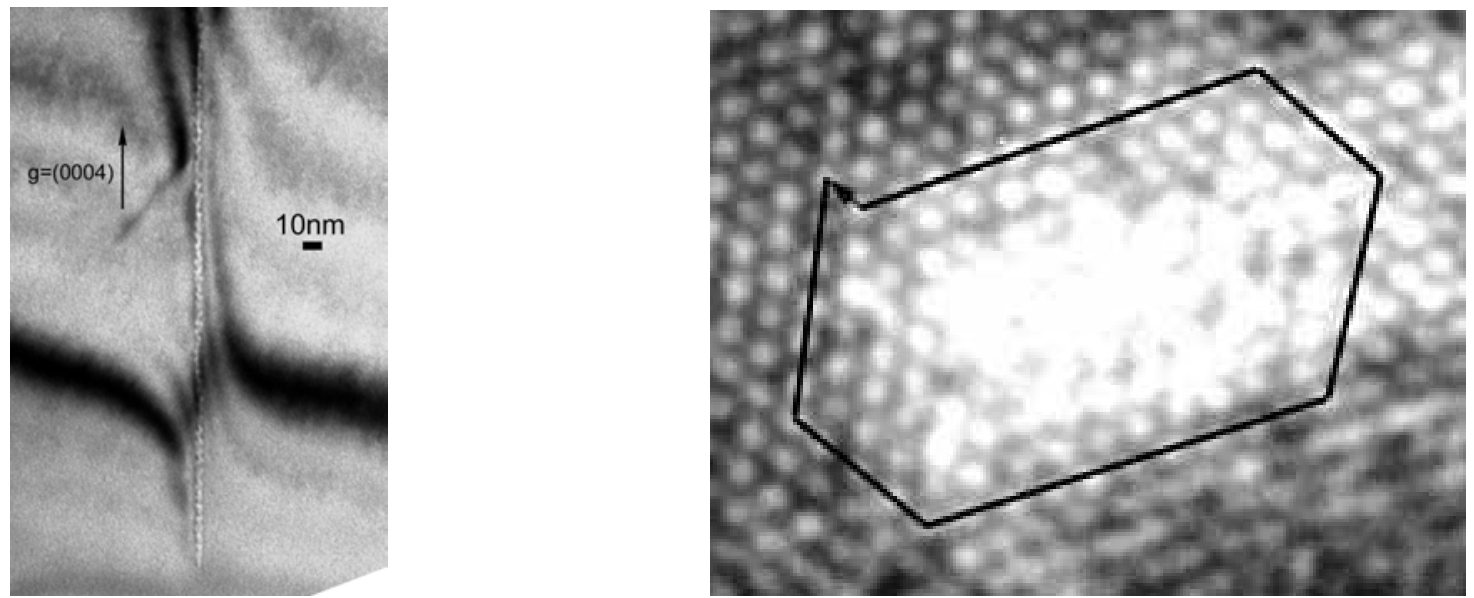

FIG. 1. Examples of hollow core dislocations. The left hand figure shows a bright field micrograph of a hollow core $c$-dislocation in HVPE-grown GaN (cross-sectional sample) crossed by a $\mathrm{g}=0004$ contour. The contour splitting, which shows three subsidiary minima is consistent with a $c$ component of Burgers vector. The right hand figure is a lattice image from a hollow core $a$ dislocation in Mg-doped GaN viewed end on (plan-view sample). The Burgers circuit reveals the $a-$ type Burgers vector (arrowed segment $=0.31 \mathrm{~nm}$ ).
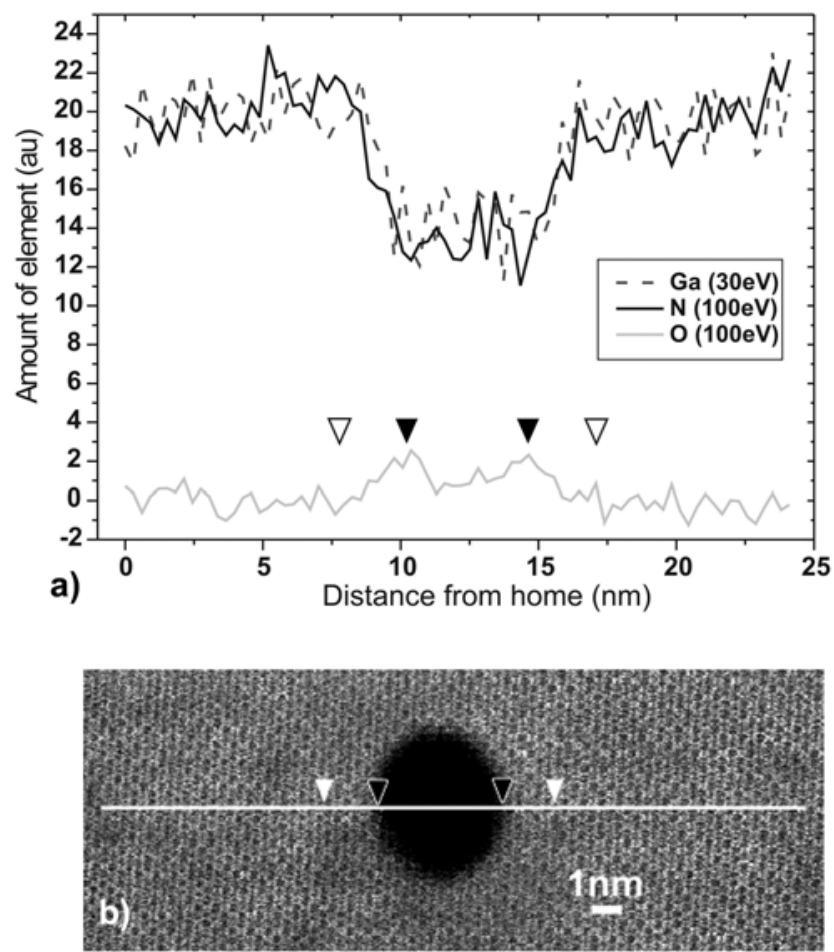

FIG. 2. (a) EELS line profiles showing how Ga, $\mathrm{N}$ and $\mathrm{O}$ vary across a hollow core dislocation in HVPE-grown GaN. The profiles were taken along the line shown in the high angle annular dark field image in (b). 